\title{
Enteroparásitos en Rattus sp "ratas" en los mercados de los distritos Tacna, Ciudad Nueva y Gregorio Albarracín - provincia Tacna, 2019
}

\author{
Enteroparasites in Rattus sp "rats" in the supply centers of the districts of Tacna, \\ Ciudad Nueva and Gregorio Albarracín of the province of Tacna, 2019
}

\author{
${ }^{1}$ Jose Félix Cruz Perca \\ ${ }^{2 *}$ Luis Lloja Lozano \\ ${ }^{3}$ Dayana Wendy Mamani Condori
}

\section{RESUMEN}

El presente trabajo de investigación se realizó en los mercados de la provincia de Tacna durante los meses de diciembre del 2018 a junio del 2019. El objetivo fue determinar los enteroparásitos presentes en las ratas de los centros de abastos. Asimismo, identificar las especies de enteroparásitos, resaltando las especies de importancia en salud pública. Se capturaron 81 roedores (79 Rattus norvegicus y 2 Rattus rattus) con trampas de captura viva (Tomahawk). Para la colecta de helmintos adultos, se empleó la técnica de Travassos. Los parásitos adultos fueron colectados, fijados con formol acético, coloreados con carmín clorhídrico, montados en láminas, e identificados, empleando técnicas convencionales. Para la observación de quistes y huevos de parásitos, se empleó el examen directo y la técnica de concentración formol-éter. Los resultados mostraron una frecuencia general de enteroparásitos de 85.19 \%. Se hallaron 15 especies de enteroparásitos: Entamoeba spp, Entamoeba coli, Iodamoeba spp, Giardia spp, Eimeria spp, Cryptosporidium spp, Hymenolepis diminuta, H. nana, Strongyloides spp, Heterakis spumosa, Aspiculuris spp, Syphacia spp, Gongylonema neoplasticum, Trichosomoides spp y Ascaris sp. Mediante la prueba de Chi cuadrado, los resultados mostraron que no existe asociación significativa entre las variables sexo, grupo etario, peso y longitud de las ratas por la infección de protozoos y helmintos.

Palabra clave: Enteroparásitos.

\section{ABSTRACT}

This research work was carried out in the markets of the province of Tacna from December 2018 to June 2019. The objective was to determine which enteroparasites are present in rats in the supply centers; likewise, identify the species of enteroparasites, highlighting the species of importance to public health. 81 rodents were captured (79 Rattus norvegicus and 2 Rattus rattus) with live catch traps (Tomahawk). Also, the Travassos Technique was used to collect adult helminths. Adult parasites were collected, fixed with Acetic Formol, stained with Hydrochloric Carmine, mounted on plates and identified, using conventional techniques. For the observation of cysts and parasite eggs, direct examination and the formaldehyde-ether concentration technique was used. Results showed an overall enteroparasite frequency of $85.19 \%$. Fifteen species of enteroparasites were found: Entamoeba spp, Entamoeba coli, Iodamoeba spp, Giardia spp, Eimeria spp, Cryptosporidium spp; Hymenolepis diminuta, H. nana, Strongyloides spp, Heterakis spumosa, Aspiculuris spp, Syphacia spp, Gongylonema neoplasticum, Trichosomoides spp, and Ascaris sp. Using the Chi-square test, the results showed that there is no significant association between the variables sex, age group, weight and length of rats due to infection with protozoa and helminth.

Keywords: Enteroparasites.

\footnotetext{
$1,2,3$ Laboratorio de Parasitología. Facultad de Ciencias. Universidad Nacional Jorge Basadre Grohmann. Tacna- Perú.

* Autor de correspondencia: lullolo1962@gmail.com
} 


\section{INTRODUCCIÓN}

Las enfermedades zoonóticas parasitarias constituyen una seria amenaza a la salud pública, por lo cual, la identificación de reservorios silvestres es de prioridad importancia. En el caso de los roedores (Rattus norvegicus y Rattus rattus), son especies que viven en estrecha asociación con las actividades humanas, como la agricultura, unidades de producción animal y viviendas. Además, son especies dominantes por su gran capacidad de adaptación a diversos ecosistemas. Asimismo, se les considera una plaga, debido a la competencia con el hombre por los mismos recursos alimenticios; contaminando los alimentos destinados al consumo humano y animal (De Sotomayor et al., 2015).

En países en desarrollo, el crecimiento urbano es desordenado con altas inequidades en salud e inadecuado saneamiento ambiental, lo que facilita la multiplicación de roedores, cobrando importancia en la cadena epidemiológica, pues actuarían como reservorios y transmisores de diversas enfermedades parasitarias en animales silvestres y domésticos, incluido el hombre (Abad et al., 2016). Los parásitos transmitidos por las ratas, en su tracto gastrointestinal, están compuestos por diferentes especies de protozoos yhelmintos.

Los centros de abastos son lugares con alta afluencia de personas; por ello, la presencia de roedores en estos sitios representa un riesgo de contaminación en el ambiente y los productos que ahí se expenden. También, aumenta el riesgo de infección en el ser humano, principalmente, por ingestión de alimentos y agua contaminados, directamente o indirectamente, con heces de roedores.

Existen reportes sobre parásitos en roedores en todo el mundo, entre los que destacan las investigaciones realizadas por Pakdel et al. (2013) en Irán, y Fagir y El-Rayah (2009) en Sudán. En América Latina, encontramos en Argentina las investigaciones de Gómez et al. (2018) y de Hancke (2016); en Guayaquil, Ecuador, a Ramírez (2018). En el Perú, son escasos las publicaciones sobre fauna parasitaria de roedores y su rol como posible transmisor o reservorio de enteroparásitos con impacto en la salud pública. Tenemos la investigación de Romero et al. (2012), De Sotomayor et al. (2015) y Abad et al. (2016), quienes reportan frecuencias de protozoos $y$ helmintos en ratas. En Tacna, los centros de abastos reúnen las condiciones necesarias para contar con la presencia de roedores, como presencia de grietas en paredes del mercado, acumulación de basura, redes de alcantarillado expuestos y la existencia de casas inhabitadas en los alrededores del mercado.

En nuestro medio, no se cuenta con un estudio sobre enteroparásitos en roedores en centros de abastos, lo que nos lleva a la necesidad de realizar la presente investigación. Asimismo, de brindar datos de los diferentes grupos de enteroparásitos, permitiendo conocer el rol que juegan los roedores y el riesgo potencial para el ser humano. Además, se busca establecer si la presencia de los parásitos intestinales, protozoos y helmintos se encuentran asociados al sexo, la edad y el peso de los roedores.

\section{MATERIAL Y MÉTODOS}

\section{Área de estudio}

El área de estudio estuvo comprendida en tres centros de abastos de la provincia de Tacna, distrito de Tacna (mercado mayorista "Miguel Grau"), distrito de Ciudad Nueva (mercado "Ciudad Nueva") y distrito de Coronel Gregorio Albarracín Lanchipa (mercado "Santa Rosa"). E1 tiempo de muestreo fue desde diciembre del 2018 hasta abril de 2019.

\section{Lugar de experimentación}

El área de procesamiento de la muestra fue el Laboratorio de Parasitología - Facultad de Ciencias de la Universidad Nacional Jorge Basadre Grohmann - Tacna.

\section{Selección de los puntos de muestreo}

Con los permisos otorgados por la autoridades provinciales y administrativos de los centros de abastos, se revisó los protocolos estandarizados de uso y cuidado de animales (Protocolo del Centro de Enfermedades Infecciosas Atlanta) (Mills, Childs, Ksiazek,y Peters, 1998). La selección de los puntos de muestreo fue no probabilista por conveniencia debido a que en Tacna no se cuenta con un estudio base sobre enteroparásitos en roedores. En la captura de roedores (Rattus norvegicus y Rattus rattus), se aplicó el método de la observación seleccionando los vertederos, desagües, periferia del mercado, 
sección comidas, sección carnes y sección verduras, que presentaban evidencia de afluencia de roedores.

\section{Muestreo}

Se consideró a cada mercado como una estación de muestreo. Siendo el mercado "Ciudad Nueva", el más pequeño; empleándose un esfuerzo de captura $\left(\mathrm{n}^{\circ}\right.$ de trampas $/ \mathrm{n}^{\circ}$ de días) de 20 jaulas Tomahawk por día durante quince (15). De este modo, se capturaron 22 roedores. Se considera este dato para los otros mercados, utilizando el mismo esfuerzo de captura (Ferrnández, 2019).

El muestreo se realizó durante 45 noches, utilizando 20 trampas de captura viva "Tomahawk", colocadas entre las 7:30 y 22:30 horas. Para ello, se prepararon cebos no tóxicos (100 g de avena con una cantidad de 20 g de maní y $5 \mathrm{ml}$ de vainilla, atún, queso frito y pellejos de pollo frito). Las trampas fueron recogidas a las 2:30 y 4:00 horas del día siguiente. Las trampas que contenían roedores fueron manipuladas cuidadosamente, para colocarlas dentro de una bolsa de polietileno con una perforación para evitar asfixia del roedor. Las bolsas fueron transportadas al Laboratorio de Parasitología Facultad de Ciencias de la Universidad Nacional Jorge Basadre Grohmann para su procesamiento, siguiendo el protocolo de bioseguridad de Mills et al. (1998). Se capturaron en vertederos, desagües y la periferia un total 81 roedores (Rattus norvegicus y Rattus rattus).

Para la obtención de la muestra, se empleó el éxito de trampeo (Índice de abundancia), definido como el número de individuos contabilizados mediante la aplicación de un esfuerzo de registro controlado.

Éxito de trampeo $=\frac{\mathrm{N}^{\circ} \text { de animales capturados }}{\text { Esfuerzo de captura }} \times 100$

Al no contar en el Laboratorio con una cabina de bioseguridad, en todo momento se aplicaron medidas de bioseguridad personal. Se utilizó vestimenta de protección (mandilones descartables estériles, mascarilla, lentes y guantes), tomándose las precauciones necesarias para reducir al mínimo la exposición con las heces y orina del roedor, evitando así la formación de aerosoles. Posteriormente, se desinfectaron el área de trabajo y los materiales empleados con lejía al $10 \%$ (contiene hipoclorito de sodio al $5 \%$ efectivo contra bacterias, virus y hongos) y, después del aseo personal de las manos, se aplicó alcohol al $70 \%$.

\section{Metodología de laboratorio}

Utilizando el equipo de protección personal (EPP), se procedió a la anestesia de los roedores. En un pedazo de algodón se roció cloroformo y se colocó dentro de una bolsa plástica, para luego usar ketamina al $10 \%$ (nombre comercial HALATAL) en una dosis de $0.1 \mathrm{ml}$ para roedores con peso menor o igual a 100 gr y para mayores de 100 gr de $0.3 \mathrm{ml}$, administrada vía intramuscular, considerando el margen terapéutico de error. Al observar al roedor inmóvil sin respuesta a estimulo, se lo coloco sobre una superficie limpia para su procesamiento.

\section{Identificación del roedor y obtención de medidas estándar}

La determinación taxonómica de los roedores se realizó por morfometría según Mills et al. (1998), Garate et al. (2011); y la identificación propuesta por Coto (2016), basada en dos características:

(1)Rasgos diferenciales interespecíficos. En Rattus norvegicus, la longitud de la cola es menor que la longitud hocico - ano y las orejas son pequeñas. Mientras que, en Rattus rattus, la longitud de la cola es mayor que la longitud hocico - ano, y sus orejas son grandes.

(2)A causa de las variaciones individuales del color, en líneas generales podemos afirmar que, Rattus norvegicus presenta, dorsalmente, un amplio espectro de variantes que van desde el marrón grisáceo o el gris puro hasta el negruzco o el marrón rojizo. El vientre es gris claro o blanco amarillento. Rattus rattus varía su tonalidad dorsal desde negro absoluto hasta marrón leonado, siendo posible hallarlo con diferentes intensidades de gris a marrón; $y$, ventralmente, comprenden el gris metálico, el gris perla o café y el blanco puro.

Para determinar el sexo de los machos, se ubicaron los testículos: y, para las hembras, la vagina (que en algunos casos no estaba expuesta a simple vista). El grupo etario se registró según el peso como juvenil y adulto. En el caso R. rattus, fueron clasificados como adultos los que tenían un peso mayor a $130 \mathrm{~g}$; mientras que, en el caso de $R$. norvegicus, los que presentaban un peso mayor de $200 \mathrm{~g}$, utilizando como referencia datos de Kataranovski et al. (2011) y Abad et al. (2016). De acuerdo al pesaje con balanza analítica, las ratas fueron divididas, según Garate et al. (2011), en categorías pesos estándar (Categoría I, con menos de $250 \mathrm{~g}$; Categoría II, con 250 g a más). Para conocer la longitud, se colocó al espécimen 
sobre una superficie con la parte ventral hacia arriba de modo que el cuerpo y la cola estén derechos, pero no estirados. Se midió la distancia desde la punta de la nariz a la punta de la parte carnosa de la cola. Posteriormente, se tomó la medida de la cola, divididas también en categorías (Categoría I, con menos de $20 \mathrm{~cm}$; Categoría II, con 20 cm a más) según Garate et al. (2011).

\section{Extracción del tubo digestivo}

Los roedores fueron sometidos a una de sobredosis de Ketamina al $10 \%$ para la eutanasia. El área de procesamiento fue desinfectada con solución de lejía al $10 \%$, según la recomendación del Instituto Nacional de Salud. Posteriormente, se realizó la disección y extracción del tubo digestivo, y fue colocado en una placa Petri con solución salina fisiológica previamente rotulada.

\section{Búsqueda de Helmintos (técnica de Travassos)}

El tubo digestivo del roedor fue humedecido constantemente con solución salina fisiológica. Posteriormente, se hizo un corte sagital al tracto digestivo. Realizada la disección, se procedió a la búsqueda exhaustiva de los helmintos empleando un estereoscopio. Los parásitos se colectaron con ayuda de pinzas finas, pinceles y estiletes, luego fueron lavados con solución salina fisiológica, para su posterior fijación.

\section{Fijación, coloración, montaje y observación de los parásitos}

Los parásitos colectados fueron sometidos al método de fijación, coloración, aclaramiento, deshidratación, montaje y observación de los parásitos, según Botero y Restrepo (2012) y Escalante (1986).

a) Con ayuda de una pinza plana, los parásitos fueron colocados sobre una lámina portaobjetos, en una gota de solución salina fisiológica.

b) Se procedió a colocar dos tiras de papel en los extremos de la lámina $\mathrm{y}$, por encima, se colocó otra lámina, se envolvió ambas láminas con pabilo.

c) Las láminas prensadas fueron colocadas en un frasco de vidrio que contenía una solución de formol acético utilizado como fijador.

d) Se retiró a los parásitos del fijador y se procedió a su lavado, colocándolos en una placa Petri que contenía agua corriente, para realizar el lavado por espacio de 1 a 2 horas. e) Los parásitos lavados fueron colocados, por 1 hora, en los colorantes Carmín clorhídrico alcohólico y hematoxilina de Dedafield. Con ayuda de un pincel, se introdujeron al fondo del colorante, evitando que tengan contacto con el aire.

f) Luego de la coloración, se lavaron los parásitos con agua destilada, retirando el exceso del colorante. Se colocaron los parásitos en alcohol-ácido para su decoloración. Luego fueron deshidratados en una batería de alcoholes de $30^{\circ}, 50^{\circ}, 60^{\circ}$, $70^{\circ}, 80^{\circ}, 90^{\circ}$ y alcohol absoluto, durante 20 minutos en cada uno.

g) Posteriormente, se colocaron los parásitos en placa Petri conteniendo xilol, por un espacio de 10 minutos.

h) El montaje se realizó con bálsamo de Canadá, utilizando láminas y laminillas bien limpias. Se agregó una gota de bálsamo sobre la lámina portaobjeto, seguidamente, se colocó al parásito con la cara ventral hacia arriba, y se cubrió cuidadosamente con la laminilla para evitar la formación de burbujas en el medio.

i) Finalmente, se dejó secar los preparados, en posición horizontal.

\section{Métodos parasitológicos}

Se colectó la mucosa intestinal en frascos conteniendo solución de formol al $5 \%$, se rotuló el frasco para su posterior observación al microscopio. Se empleó la técnica de observación directa, la técnica de concentración formol - éter y la coloración con Ziehl Neelsen, modificada para Cryptosporidium.

Concentración formol - éter: Procedimiento (MINSA, 2003):

a) Se homogenizó una porción de la muestra de la mucosa intestinal, preparando una suspensión de $10 \mathrm{~g}$ de la muestra en $90 \mathrm{ml}$ de agua destilada, en cada tubo de ensayo.

b) Se filtró la suspensión a través de un colador o una gasa doblada en cuatro, en un recipiente limpio, colocando en un tubo de ensayo la mezcla filtrada.

c) Se centrifugó el filtrado a $1500 \mathrm{rpm}$ por 3 min, decantando el líquido sobrenadante. Al sedimento, se completó con solución de formol al $10 \%$ hasta igualar la medida anterior. Se homogenizó y se dejó reposar por 5 minutos, para luego adicionar $3 \mathrm{ml}$ de éter.

d) Se tapó el tubo y se procedió a agitarlo suavemente, luego, se eliminaron las capas formadas de sobrenadante. 
e) Se volvió a centrifugar a $3000 \mathrm{rpm}$ durante 3 minutos. Luego, se decantó el líquido sobrenadante. Del sedimento, con un gotero, se extrajo una gota de la muestra, colocándola en una lámina porta objeto a la cual se adicionó una gota de lugol parasitológico. Se observó el resultado, a través del microscopio.

\section{Determinación taxonómica}

La determinación taxonómica de las especies parásitas se realizó a partir de la observación microscópica, mediante fotos, y empleando las claves taxonómicas descritas por Cabrera y Mendoza (2001), Soulsby (1987) y Suckow et al. (2001).

\section{Análisis estadístico}

Se realizó un análisis descriptivo de la infección por enteroparásitos en ratas, determinando la frecuencia para todos los parásitos encontrados. La frecuencia de infección se determinó por el número de animales parasitados entre el número de animales examinados en un tiempo determinado expresado en porcentaje.
Frecuencia $=\frac{\text { Número de animales parasitados }}{\text { Número de animales examinados }} \times 100$

Se ingresaron los datos a la herramienta de datos estadísticos Excel 2016, donde se construyeron gráficas de frecuencia para señalar cantidades y porcentajes. Se realizó el test de comparación de proporciones basado en la distribución $\mathrm{X}^{2}$ con una asociación significativa ( $>0,05)$, empleando el programa de análisis estadístico InfoStat.

\section{RESULTADOS}

Determinación de la frecuencia de enteroparásitos en Rattus sp en centros de abastos de la provincia de Tacna

De un total de 81 roedores capturados, 79 especímenes $(97.53 \%)$ corresponden a Rattus norvegicus. De esta cantidad, 22 especímenes $(27.16 \%)$ fueron capturados en el mercado "Ciudad Nueva"; 25 (30.86 \%), en el mercado "Santa Rosa"; y 32 (39.51\%), en el mercado "Miguel Grau". Por otro lado, de la especie Rattus rattus, se capturaron 2 especímenes $(2.47 \%)$ en el mercado "Miguel Grau", tal como se muestra en la Tabla 1.

Tabla 1. Especies de Rattus norvergicus y Rattus rattus capturados, según los mercados de abastos en la provincia de Tacna.

\begin{tabular}{lcccccc} 
& \multicolumn{2}{c}{ Rattus norvegicus } & \multicolumn{2}{c}{ Rattus rattus } & \multicolumn{2}{c}{ Total } \\
\cline { 2 - 7 } Mercado & $\mathbf{n}$ & $\mathbf{\%}$ & $\mathbf{n}$ & $\mathbf{\%}$ & $\mathbf{n}$ & $\mathbf{\%}$ \\
\hline Ciudad & 22 & 27.16 & - & - & 22 & 27.16 \\
Nueva & 25 & 30.86 & - & - & 25 & 30.86 \\
Santa Rosa & 32 & 39.51 & 2 & $2.47 \%$ & 34 & 41.98 \\
Miguel Grau & & &
\end{tabular}

Como se muestra en la Tabla 2, se encontraron parásitos protozoos y/o helmintos en $85.19 \%$ del total de roedores capturados. Por otro lado, se encontró que el 14.81 \% no mostró infección por enteroparásitos.

Tabla 2. Frecuencia general de infección por enteroparásitos en Rattus sp.

\begin{tabular}{lcc} 
& \multicolumn{2}{c}{$\begin{array}{c}\text { Frecuencia de Infección por } \\
\text { Enteroparásitos }\end{array}$} \\
\cline { 2 - 3 } Ratas & $\mathbf{n}$ & $\mathbf{\%}$ \\
\hline Parasitados & 69 & 85.19 \\
No Parasitados & 12 & 14.81 \\
\hline Total & 81 & 100
\end{tabular}

La frecuencia parasitaria fue de $60.49 \%$ para protozoos, y de $74.07 \%$ para helmintos (Tabla 3 ). 
Tabla 3. Frecuencia de infección por protozoos y helmintos en Rattus sp

\begin{tabular}{lcccc} 
& $\begin{array}{c}\text { Frecuencia de infección por } \\
\text { protozoos }\end{array}$ & $\begin{array}{c}\text { Frecuencia de infección } \\
\text { por helmintos }\end{array}$ \\
\cline { 2 - 5 } & $\mathbf{N}$ & $\mathbf{\%}$ & $\mathbf{N}$ & $\mathbf{\%}$ \\
\hline Parasitados & 49 & 60.49 & 60 & 74.07 \\
No parasitados & 32 & 39.51 & 21 & 26.93 \\
\hline Total & 81 & 100 & 81 & 100 \\
\hline
\end{tabular}

Los protozoos, en mayor frecuencia, corresponden a Cryptosporidium spp (28.39 \%) y a Entamoeba spp $(27.16 \%)$. Por otro lado, en menor frecuencia, corresponden a Entamoeba coli (7.41\%) y a Iodamoeba spp $(1.23 \%)$ (Tabla 4$)$.

Tabla 4. Enteroparásitos protozoos identificados en Rattus sp, en centros de abastos de la provincia de Tacna

\begin{tabular}{|c|c|c|c|c|c|c|c|c|}
\hline \multirow{2}{*}{ ENTEROPARÁSITOS } & \multicolumn{2}{|c|}{$\begin{array}{c}\text { Total de ratas } \\
\text { infectadas } \\
\end{array}$} & \multicolumn{2}{|c|}{$\begin{array}{c}\text { Mercado Miguel } \\
\text { Grau }\end{array}$} & \multicolumn{2}{|c|}{$\begin{array}{c}\text { Mercado Santa } \\
\text { Rosa }\end{array}$} & \multicolumn{2}{|c|}{$\begin{array}{c}\text { Mercado Ciudad } \\
\text { Nueva }\end{array}$} \\
\hline & $\mathbf{n}=\mathbf{8 1}$ & $\%$ & $\mathrm{n}=\mathbf{3 4}$ & $\%$ & $\mathbf{n}=\mathbf{2 5}$ & $\%$ & $\mathbf{n}=\mathbf{2 2}$ & $\%$ \\
\hline \multicolumn{9}{|l|}{ Protozoos } \\
\hline Entamoeba spp* & 22 & 27.16 & 14 & 41.17 & 5 & 20 & 3 & 13.63 \\
\hline Entamoeba coli ${ }^{*}$ & 6 & 7.41 & 3 & 8.82 & - & - & 3 & 13.63 \\
\hline Iodamoeba spp* & 1 & 1.23 & - & - & - & - & 1 & 4.54 \\
\hline Giardia spp* & 13 & 16.05 & 5 & 14.70 & 5 & 20 & 3 & 13.63 \\
\hline Eimeria spp & 15 & 18.52 & 5 & 14.70 & 4 & 16 & 6 & 27.27 \\
\hline Cryptosporidium spp* & 23 & 28.39 & 9 & 26.47 & 6 & 24 & 8 & 36.36 \\
\hline
\end{tabular}

(*) Enteroparásitos de importancia zoonótica

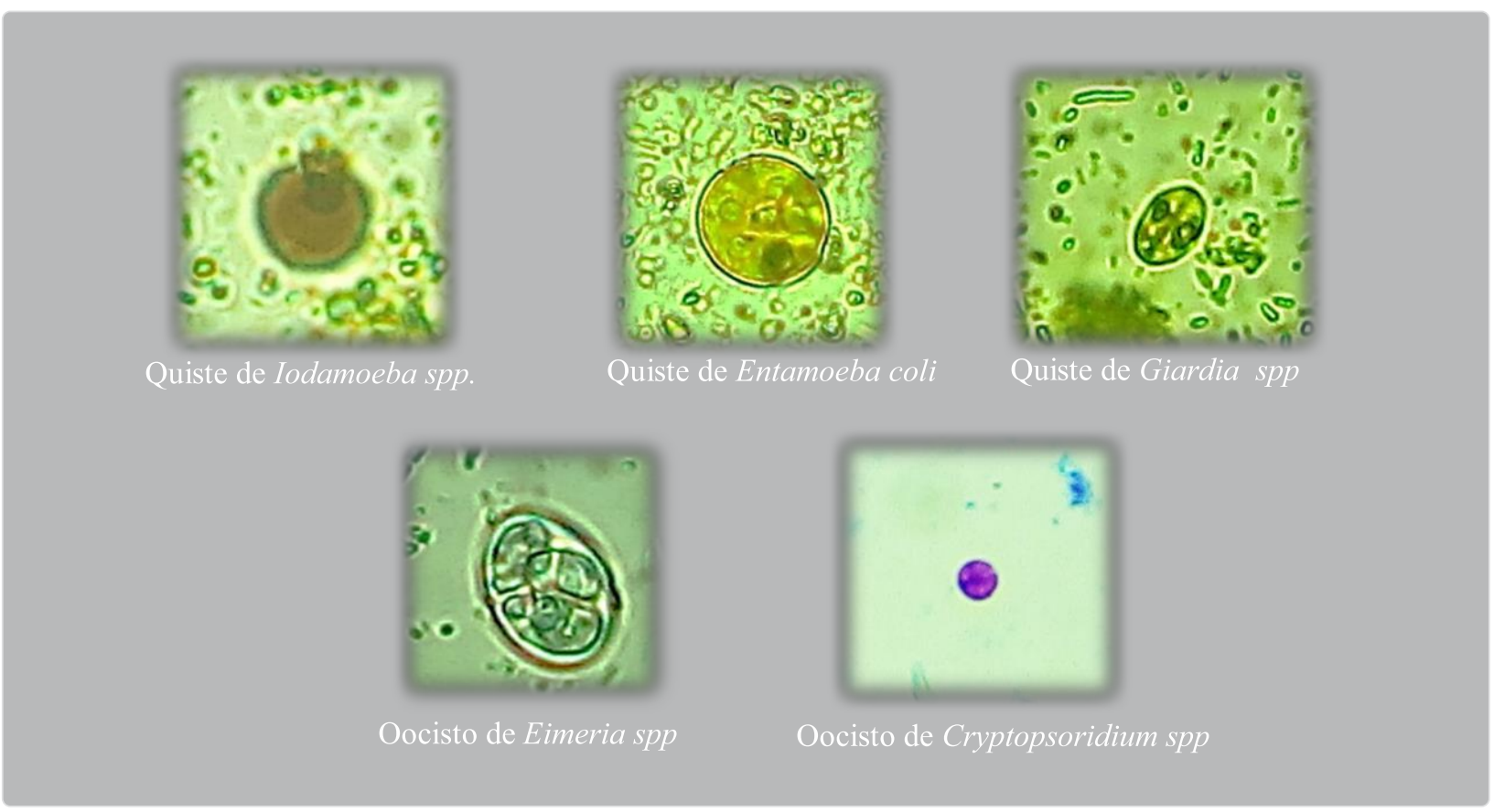

Figura 1. Formas evolutivas de protozoos observados con microscopio óptico en muestras de Rattus sp

En el caso de los helmintos, se detectó a Heterakis spumosa en un $33.33 \%$, nematodo que se encontró en mayor frecuencia. Los cestodos Hymenolepis diminuta (24.69 \%) y Hymenolepis nana (17.28 \%), así como los nematodos Strongyloides spp (18.52 \%) y Gongylonema neoplasticum (14.81 \%) fueron los helmintos de importancia zoonótica que presentaron una frecuencia de infección alta (Tabla 5) (Fig. 2). 
Enteroparásitos en Rattus sp "ratas" en los mercados de los distritos Tacna, Ciudad Nueva y Gregorio Albarracín - provincia Tacna, 2019

Tabla 5. Enteroparásitos helmintos identificados en Rattus sp en centros de abastos de la provincia de Tacna.

\begin{tabular}{|c|c|c|c|c|c|c|c|c|}
\hline \multirow[t]{2}{*}{ ENTEROPARÁSITOS } & \multicolumn{2}{|c|}{$\begin{array}{c}\begin{array}{c}\text { Total de ratas } \\
\text { infectadas }\end{array} \\
\end{array}$} & \multicolumn{2}{|c|}{$\begin{array}{c}\text { Mercado Miguel } \\
\text { Grau }\end{array}$} & \multicolumn{2}{|c|}{$\begin{array}{c}\text { Mercado Santa } \\
\text { Rosa }\end{array}$} & \multicolumn{2}{|c|}{$\begin{array}{c}\text { Mercado Ciudad } \\
\text { Nueva }\end{array}$} \\
\hline & $\mathrm{n}=\mathbf{8 1}$ & $\%$ & $\mathrm{n}=\mathbf{3 4}$ & $\%$ & $\mathrm{n}=\mathbf{2 5}$ & $\%$ & $\mathrm{n}=\mathbf{2 2}$ & $\%$ \\
\hline \multicolumn{9}{|l|}{ Helmintos } \\
\hline Hymenolepis diminuta* & 20 & 24.69 & 7 & 20.59 & 9 & 36 & 4 & 18.18 \\
\hline Hymenolepis nana* & 14 & 17.28 & 6 & 17.65 & 5 & 20 & 3 & 13.63 \\
\hline Strongyloides spp* & 15 & 18.52 & 5 & 14.70 & 3 & 12 & 7 & 31.82 \\
\hline Heterakis spumosa & 27 & 33.33 & 15 & 44.12 & 7 & 28 & 5 & 22.27 \\
\hline Syphacia spp & 7 & 8.64 & 3 & 8.82 & - & - & 4 & 18.18 \\
\hline Gongylonema neoplasticum* & 12 & 14.81 & 5 & 14.70 & 5 & 20 & 2 & 9.09 \\
\hline Aspiculuris spp & 3 & 3.70 & - & - & - & - & 3 & 13.63 \\
\hline Trichosomoides spp & 1 & 1.23 & 1 & 2.94 & - & - & - & - \\
\hline Ascaris spp* & 2 & 2.47 & 1 & & - & - & 1 & 4.54 \\
\hline
\end{tabular}

(*) Enteroparásitos de importancia zoonotica

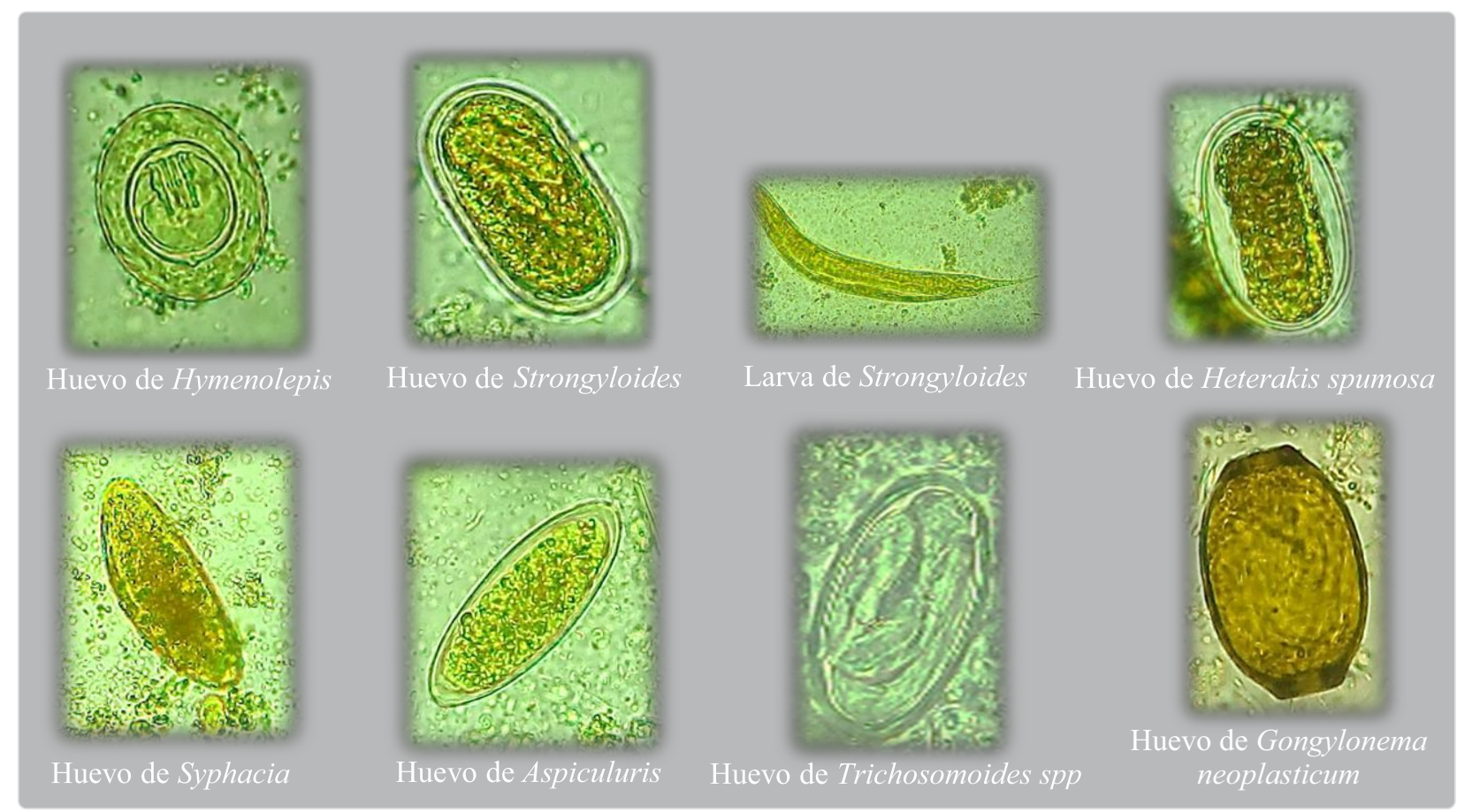

Figura 2. Formas evolutivas de Helmintos observados con microscopio óptico en muestras de Rattus sp

Al observar la relación entre las variables sexo, grupo etario, peso y longitud del roedor frente a los protozoos, mediante la prueba del Chi Cuadrado $\mathrm{X}^{2}$ con un nivel de significancia de 0.05 , se encontró que no existe asociación significativa (Tabla 6). 
Tabla 6. Relación entre la infección por protozoos y las variables sexo, grupo etario, peso y longitud del roedor

\begin{tabular}{|c|c|c|c|c|c|c|}
\hline \multirow[t]{2}{*}{ Variable } & & \multirow{2}{*}{$\begin{array}{l}\text { Muestreadas } \\
\qquad(\mathrm{n}=\mathbf{8 1})\end{array}$} & \multicolumn{2}{|c|}{$\begin{array}{c}\text { Ratas con infección } \\
\text { por helmintos }\end{array}$} & \multirow[t]{2}{*}{$\mathbf{X}^{2}$} & \multirow[t]{2}{*}{$\mathbf{P}$} \\
\hline & & & $n=60$ & $\%$ & & \\
\hline \multirow[t]{2}{*}{ Sexo } & Macho & 42 & 31 & 73.81 & \multirow{2}{*}{0.003} & \multirow{2}{*}{0.95} \\
\hline & Hembra & 39 & 29 & 74.36 & & \\
\hline \multirow[t]{2}{*}{ Grupo etario } & Juvenil & 37 & 26 & 70.27 & \multirow{2}{*}{0.513} & \multirow{2}{*}{0.473} \\
\hline & Adulto & 44 & 34 & 77.27 & & \\
\hline \multirow[t]{2}{*}{ Peso } & Categoría I & 42 & 31 & 73.81 & \multirow{2}{*}{0.003} & \multirow{2}{*}{0.955} \\
\hline & Categoría II & 39 & 29 & 74.36 & & \\
\hline \multirow[t]{2}{*}{ Longitud } & Categoría I & 59 & 44 & 74.58 & \multirow{2}{*}{0.028} & \multirow{2}{*}{0.865} \\
\hline & Categoría II & 22 & 16 & 72.73 & & \\
\hline
\end{tabular}

Peso (Categoría I = menor a 250 g y Categoría II = 250 g a más). Longitud (Categoría I = menor de $20 \mathrm{~cm}$ y Categoría II $=20 \mathrm{~cm}$ a más).

Al observar la relación entre las variables sexo, grupo etario, peso y longitud del roedor frente helmintos encontrados, mediante la prueba del Chi Cuadrado $\mathrm{X}^{2}$ con un nivel de significancia de 0.05 , se concluye que no existe asociación significativa (Tabla 7).

Tabla 7. Relación entre la infección por helmintos y las variables sexo, grupo etario, peso y longitud

\begin{tabular}{|c|c|c|c|c|c|c|}
\hline \multirow[t]{2}{*}{ Variable } & & \multirow{2}{*}{$\begin{array}{l}\text { Muestreadas } \\
\qquad(\mathrm{n}=\mathbf{8 1})\end{array}$} & \multicolumn{2}{|c|}{$\begin{array}{l}\text { Ratas con infección } \\
\text { por protozoos }\end{array}$} & \multirow[t]{2}{*}{$X^{2}$} & \multirow[t]{2}{*}{$\mathbf{P}$} \\
\hline & & & $n=49$ & $\%$ & & \\
\hline Sexo & $\begin{array}{l}\text { Macho } \\
\text { Hembra }\end{array}$ & $\begin{array}{l}42 \\
39\end{array}$ & $\begin{array}{l}26 \\
23\end{array}$ & $\begin{array}{l}61.90 \\
58.97\end{array}$ & 0.07 & 0.787 \\
\hline Grupo etario & $\begin{array}{l}\text { Juvenil } \\
\text { Adulto }\end{array}$ & $\begin{array}{l}37 \\
44\end{array}$ & $\begin{array}{l}21 \\
28\end{array}$ & $\begin{array}{l}56.76 \\
63.64\end{array}$ & 0.40 & 0.528 \\
\hline Peso & $\begin{array}{l}\text { Categoría I } \\
\text { Categoría II }\end{array}$ & $\begin{array}{l}42 \\
39\end{array}$ & $\begin{array}{l}22 \\
27\end{array}$ & $\begin{array}{l}52.38 \\
69.23\end{array}$ & 2.40 & 0.121 \\
\hline Longitud & $\begin{array}{l}\text { Categoría I } \\
\text { Categoría II }\end{array}$ & $\begin{array}{l}59 \\
22\end{array}$ & $\begin{array}{l}37 \\
12\end{array}$ & $\begin{array}{l}62.71 \\
54.55\end{array}$ & 0.45 & 0.503 \\
\hline
\end{tabular}

Peso (Categoría I = menor a 250 g y Categoría II = 250 g a más). Longitud (Categoría I = menor de $20 \mathrm{~cm}$ y Categoría II $=20 \mathrm{~cm}$ a más).

\section{DISCUSIÓN}

Investigaciones realizadas por Abad et al. (2016), De Sotomayor et al. (2015) e Iannacone y Alvariño (2002) reportan mayor presencia de Rattus rattus, a diferencia de los resultados que se muestran en la Tabla 1, donde se registra a Rattus norvegicus en mayor cantidad. Esta diferencia se debería a que dichos autores realizaron el muestreo en un área de estudio diferente y diverso (viviendas, camales, zoológicos, áreas agrícolas, chancherías, etc.). Abad et al. (2016) considera que la especie $R$. rattus se asocia típicamente con la presencia de basura. Además, atraída por los alimentos, puede ser encontrada en bodegas, restaurantes y mercados, ya que esta especie es considerada buena trepadora y tiende a habitar en lugares elevados, desplazándose a través de los cables y falsos techos de los mercados. En nuestro estudio, los mercados de Tacna presentaron condiciones favorables para la propagación de $R$. norvegicus o rata de alcantarilla. Las deficiencias en los desagües, huecos en las paredes y casas abandonadas en zonas aledañas al mercado permitieron que esta especie se desarrolle mejor que $R$. rattus. Otro factor importante fue la presencia de lluvias y el colapso de desagües en los mercados y en la ciudad. Por otro lado, la 
frecuencia significativa de gatos observados en los techos de los mercados, influyó considerablemente en la captura de esta especie. Según Hancke (2016), considera que $R$. norvegicus es una especie dominante en ambientes con un grado de urbanización baja y media.

Coto (2016) menciona que, en las poblaciones de ratas, el número de individuos de un área determinada se ve afectado por los indicadores: natalidad y mortalidad, emigración e inmigración. Estos factores, a su vez, son directamente influenciados por una serie de elementos bióticos y abióticos. Entre ellos, destacan la disponibilidad de alimentos y refugio, y las relaciones inter e intraespecíficas. El género Rattus se ajusta a un modelo de dinámica poblacional denso dependiente. Es decir, las variables que controlan las tasas de natalidad y mortalidad, emigración e inmigración son consecuencia directa de la densidad poblacional.

En la salud pública, $R$. rattus y $R$. norvegicus participan en el ciclo de infección de numerosas enfermedades zoonóticas, como reservorios naturales, hospederos intermediarios e, incluso, como hospederos de los ectoparásitos vectores transmisores de patógenos. Los agentes etiológicos transmitidos a los seres humanos son mediante dos vías: (1) directa, cuando la infección es consecuencia de mordidas o ingesta de alimentos contaminados con desechos (saliva, heces u orina) de individuos infectados, e (2) indirecta, cuando la infección es realizada por contacto o manipulación de superficies, alimentos contaminados o por inhalación de microorganismos presentes en las heces fecales como en el caso de los Hantavirus (Torres, 2017).

La frecuencia de infección parasitaria que se muestra en la Tabla 2, se asemeja al trabajo de Ayulo y Dammert en Lima (1947), donde se reporta una frecuencia general de $87.30 \%$. La gran diversidad parasitaria obtenida en este estudio podría deberse a las actividades realizadas dentro de los mercados y a una proliferación de hospederos intermediarios, esto refleja el grado de hacinamiento que se da en los mercados, pudiendo aumentar la cantidad de ratas infectadas. Según Hancke (2016), en ambientes muy heterogéneos dentro del área de acción de un hospedador, la explotación de los recursos disponibles tiende a ser más diversificada, incrementando la infección con una mayor cantidad de especies de parásitos. La frecuencia de infección para protozoos (Tabla 3) es mayor a lo reportado por Ayulo y Dammert (1947), esto debido a la simplicidad de infección y el ciclo biológico que desarrollan, con básicamente dos formas evolutivas en su ciclo de vida, encontrándose en ella a los quistes como una forma de resistencia a las condiciones medioambientales adversas.

La frecuencia de infección para helmintos, reportada en la Tabla 3, es similar a los estudios de Abad et al. (2016), pero menor a la frecuencia de infección por De Sotomayor et al. (2015), Iannacone y Alvariño (2002), Archer et al. (2017) y Suarez y Valencia (1986). Por otro lado, se encuentra por encima de las registradas en Ecuador(Ramirez, 2018), en Argentina (Gómez et al., 2018), en Tailandia (Chaisiri et al, 2018) y en Sudán (Fagir y El-Rayah, 2009). Se debe considerar que los reportes mencionados no fueron realizados necesariamente en mercados de abastos, y esto se evidencia en la riqueza parasitaria y la baja abundancia en nuestros reportes. Esto, probablemente, se deba a la presencia de hospedadores intermediarios infectados, el sistema inmunológico de las ratas y su baja densidad poblacional (Abad et al., 2016).

En la Tabla 4, se reportan 6 especies de protozoos: Entamoeba coli, Iodamoeba spp, Giardia spp, Eimeria spp, Entamoeba spp y Cryptosporidium spp. Siendo las dos últimas, las especies encontradas con mayor frecuencia. Estos resultados pueden atribuirse a una elevada contaminación ambiental y al mecanismo de transmisión por via oral - fecal; debido a que la rata de alcantarilla ( $R$. norvegicus) se encuentra en contacto con heces humanas y de animales, así como posibles restos vegetales contaminados en el desagüe de los mercados. Ayulo y Dammert (1947) consideran que la rata posee una importancia muy pequeña como reservorio de amebiasis humana. Así mismo, Eimeria spp presenta una frecuencia relativamente alta en el intestino de las ratas, considerando a este protozoo eurixeno, por su especificidad, bien adaptado a su hospedero. Esto, probablemente, se deba al tiempo corto de esporulación que presenta cuando las condiciones ambientales son favorables, tal como se observa en los mercados materia de estudio. En roedores silvestres, se ha informado que la coccidiosis causa colitis y la reproducción en animales juveniles (Suckow et al., 2001). Del mismo modo, Giardia spp se 
reporta con una alta frecuencia (Ayulo y Dammert, 1947; Casana, 2018). Vives y Zeledón (1957) también lo mencionan, pero no establecieron su frecuencia. Estos roedores albergan a Giardia muris y Giardia lamblia. La alta frecuencia reportada en este trabajo es un indicativo que las medidas sanitarias son mínimas en los mercados de la provincia de Tacna, permitiendo así condiciones para la transmisión de estos parásitos.

En la Tabla 5, se reportan nueve especies de helmintos; entre ellos, dos especies de cestodos (Hymenolepis diminuta e Hymenolepis nana) y siete especies de nematodos (Strongyloides spp, Heterakis spumosa, Syphacia spp, Gongylonema neoplasticum, Aspiculuris spp, Trichosomoides spp y Ascaris spp).

La frecuencia de Hymenolepis diminuta es mayor que la reportada por Ramírez (2018), y menor a lo reportado por De Sotomayor et al (2015), Abad et al. (2016) y a Iannacone y Alvariño (2002). La presencia de este parásito en los roedores es un indicativo para afirmar que se ha reforzado su papel como reservorio (De Sotomayor, 2015), observándose un incremento de hospedadores intermediarios en los mercados de la provincia de Tacna, siendo de los géneros Tenebrio y Tribolium. Este helminto es cosmopolita; trabajos realizados en el mundo consideran a la rata como su reservorio natural. Se observa también su potencial zoonótico, pudiendo infectar a los humanos, especialmente a los niños. Garate et al. (2011) constituyen el primer registro del rol de Xenopylla cheopis como hospedero intermediario natural en Perú. Por otro lado, la frecuencia de infección de Hymenolepis nana es mayor que la determinada por De Sotomayor et al (2015) y Abad et al (2016), pero menor que la determinada por Ramírez (2018). Es una especie reportada en diferentes partes del mundo, y muy estudiada por ser de interés zoonótico. La alta frecuencia de este parásito en roedores es un indicativo de que personas y animales se encuentran infectados con H. nana, en los mercados de la provincia de Tacna. Abad et al. (2016) mencionan que estudios recientes han demostrado que las cepas que infectan roedores y las que infectan humanos son diferentes. Sin embargo, no se debe ignorar el potencial zoonótico, debido a que sus ciclos biológicos son semejantes.

En los nematodos, Heterakis spumosa fue el helminto de mayor frecuencia al contrastar los resultados con De Sotomayor et al. (2015), pero obtuvo una menor frecuencia según lo reportado por Ramírez (2018). Según Cabrera y Mendoza (2001), es común encontrar este parasito en múridos, y su amplia distribución geográfica es porque presenta con un ciclo de vida directo (ciclo monoxeno), esto favorece la infección en las ratas. En el Perú, fue reportado por primera vez por Ayulo y Dammert (1947) y fue redescrito en Ica por Cabrera y Mendoza (2001). Además, fue incluido en la lista de parásitos de múridos de Chávez \& Zaldívar (1967), Zaldívar (1991), Sarmiento et al. (1999), Ibáñez (1966), Tantalean (1976) y Martínez y Tantalean (1998).

La frecuencia de infección expresado en porcentaje de Strongyloides spp es mayor que la determinada por Ramírez (2018), pero menor que la determinada por De Sotomayor et al (2015). Los autores reportan a la especie Stongyloides ratti como un nematodo común en los múridos, que se extiende en un amplio rango geográfico; por ello, en las regiones, las coinfecciones son muy comunes (Ramírez, 2018). Además, al encontrarse en ratas y dada la posibilidad de infestación en humanos, deben considerarse a los roedores como reservorios de este parásito.

Resultados variados sobre la presencia de Gongylonema neoplasticum han sido reportados en ratas por De Sotomayor et al. (2015) y Abad et al. (2016). Se le considera un nematodo cosmopolita, y presenta un ciclo de vida indirecto; además, tiene como hospederos intermediarios a escarabajos y cucarachas. El bajo porcentaje reportado en este estudio pudo deberse a programas de desratización y limpieza de los mercados, durante los meses anteriores, disminuyendo los hospedadores intermediarios y reduciendo la cantidad de ratas infectadas. Del mismo modo, existen resultados variados de la frecuencia de infección de Syphacia spp, obtenidos por De Sotomayor et al. (2015), Iannacone y Alvariño (2002) y Abad et al. (2016), siendo las especies encontradas $S$. muris y $S$. obvelata. Es común encontrar este parásito en ratas de pastizales. Por otro lado, la baja frecuencia de este parásito, obtenido en este estudio, pudo deberse a la ingesta de vegetales poco contaminados. Este parásito presenta un gran dimorfismo sexual, siendo el macho más pequeño que la hembra, por lo que se suele considerar a la hembra en índices de infección de ambas especies (Hancke, 2016). La baja densidad poblacional de roedores en mercados evidencia la 
baja abundancia de parásitos intestinales encontrados en este estudio, considerando que un gran número de individuos genera un mayor hacinamiento y mayores probabilidades de infección por enteroparásitos (Abad etal., 2016). La frecuencia de infección de Aspiculuris spp es menor a la reportada por De Sotomayor et al. (2015), y Abad et al. (2016), habiéndose encontrado a la especie A. tetráptera en los reportes de los investigadores en mención. Esta especie presenta un ciclo de vida directo, con una transmisión boca-anal, y es frecuente en ratas. En este estudio se reporta una baja frecuencia, probablemente se deba a una fuerte respuesta inmune por parte del hospedador.

La frecuencia de infección por Trichosomoide spp es baja, respecto a los estudios de Vives y Zeledon (1957). La baja frecuencia registrada se debe posiblemente a su localización en la vejiga urinaria y uréteres de los roedores; las larvas suelen entrar a la pared del estómago y penetrar en los vasos sanguíneos (Mehllhorn, 2016). En Perú, también fue reportada, por Tantalean (1976), en $R$. rattus, y nosotros la reportamos en $R$. norvegicus. La frecuencia de infección de Ascaris spp es baja, no se ha encontrado reportes de esta especie en Rattus sp en Perú. Archer et al. (2017) sí lo reporta para South África. La baja frecuencia en este estudio permite afirmar que Rattus norvegicus no cumple el papel de reservorio para Ascaris spp. Es importante señalar que, este enteroparásito está relacionado a la contaminación y deficiencias de saneamiento en los mercados de la provincia de Tacna. Hancke (2016) plantea que "una de las estrategias que caracteriza a los parásitos invasores es su potencialidad de poder de infectar a un amplio rango de especies de hospedadores a fin de incrementar su supervivencia aun en casos de extinción o erradicación de la especie de hospedador original".

Las especies parasitas no se encuentran distribuidas aleatoriamente, sino que la estructura de las comunidades parásitas está influenciada por diversos factores, como son las características propias del hospedador, su tamaño corporal, su longevidad, distribución geográfica y densidad poblacional. Son estos factores, los que determinan la riqueza específica de sus parásitos (Hancke, 2016). Para la primera relación de la infección por protozoos con las variables sexo, grupo etario, peso y longitud de las ratas (Tabla 6), se destaca las frecuencias, expresado en porcentajes con la infección por protozoos, observando una mínima diferencia de proporciones en todas las variables, con excepción de la variable peso (Categoría $\mathrm{I}=52.38$ $\%$ y la Categoría II $=69.23 \%$ ), obteniendo una diferencia de $16.85 \%$. Ayulo y Dammert (1947) consideran la dieta como un aspecto muy importante en la infección de las ratas con diferentes protozoos. Además, al compartir el mismo hábitat y poseer fases evolutivas resistentes a las condiciones ambientales como los quistes, estos roedores se pueden infectar en cualquier etapa de su vida. Mediante la prueba Chi cuadrado $\left(\mathrm{X}^{2}\right)$, utilizando un intervalo de confianza al $95 \%$, estadísticamente no se encontró ninguna asociación entre las variables sexo $(p=0.787)$, edad $(p=0.528)$, peso $(p=$ $0.121)$ y longitud $(\mathrm{p}=0.503)$ frente a la infección por protozoos, obteniendo valores " $p$ " mayores de 0.05 .

Para la segunda relación de la infección por helmintos, respecto a las variables sexo, edad, peso y longitud de las ratas (Tabla 7), también destacan frecuencias expresadas en porcentajes con la infección por helmintos, obteniendo diferencias casi imperceptibles en la mayoría de las variables. Sobre la variable edad (juvenil = $70.27 \%$ y adulto $=77.27 \%$ ), se observa una diferencia mínima de 7 \%. Garate et al. (2011) sugiere que, a mayor edad en las ratas, mayor será el consumo de hospederos intermediarios a lo largo de su vida, aumentando las probabilidades de infección por helmintos. Con respecto al sexo ( macho $=73.81 \%$ y hembra $=74.36 \%$ ), no se ha encontrado diferencias de proporciones. Rattus norvegicus, con respecto a su alimentación y consumo de agua, ambos sexos (macho y hembra) se encuentran expuestos a la infección por parásitos helmintos, en los mercados de la provincia de Tacna. Iannacone y Alvariño (2002) señalan que, al no existir diferencias cuantitativas en relación al sexo del huésped, se considera que no existen diferencias en la biología y la dinámica poblacional entre huéspedes machos y hembras. En el peso (Categoría $\mathrm{I}=73.81 \%$ y Categoría $\mathrm{II}=$ $74.36 \%)$ de las ratas, tampoco se evidenció diferencias de proporciones. La gran diversidad parasitaria de helmintos, respecto a la variable peso de las ratas, demuestra la adaptabilidad y capacidad que poseen para soportar el parasitismo. En la longitud (Categoría $\mathrm{I}=74.58 \%$ y Categoría II $=72.73 \%$ ) de las ratas, no se observan diferencias en las proporciones. Si bien, a mayor longitud se encontró mayor diversidad de helmintos, la infección en ambos es similar. Esto, 
probablemente, esté relacionado a su comportamiento alimentario $\mathrm{y}$ al hábitat que comparten con otros individuos, lo que permite su exposición a la infección por diversos helmintos. Si se muestra una elevada infección a helmintos, la longitud está relacionado a la edad del roedor, y la exposición a hospederos intermediarios. Mediante la prueba Chi cuadrado $\left(\mathrm{X}^{2}\right)$, utilizando un intervalo de confianza al $95 \%$, estadísticamente no se encontró ninguna asociación entre las variables sexo $(\mathrm{p}=0.955)$, edad $(p=0.473)$, peso $(p=0.955)$ y longitud $(p=$ 0.865 ) frente a la infección por helmintos, obteniendo valores "p" mayores de 0.05 .

\section{CONCLUSIONES}

Se determinó una elevada frecuencia parasitaria en ratas capturadas en los mercados de los distritos de Tacna, Ciudad Nueva y Gregorio Albarracín de la provincia de Tacna.

Se identificaron seis especies de protozoos: Entamoeba spp, Entamoeba coli, Iodamoeba spp, Giardia spp, Eimeria spp y Cryptosporidium spp. Asimismo, se identificaron nueve especies de helmintos: Hymenolepis diminuta, Hymenolepis nana, Heterakis spumosa, Strongyloides spp, Gongylonema neoplasticum, Syphacia spp, Aspiculuris spp, Trichosomoides spp y Ascaris spp,

No existen asociaciones significativas en las variables sexo, grupo etario, peso y longitud de las ratas con la infección por protozoos y helmintos.

\section{REFERENCIAS}

Abad. D, Chavez. A, Pinedo. R, Tantalean. M. y Gonzales. O. (2016). Helmintofauna gastrointestinal de Importancia zoonotica y sus aspectos en roedores (Rattus spp) en tres medioambientes. Rev Investig. Vet, 27(4), 737-747.

Archer, C. Appleton, C. Mukaratirwa, S. Lamb, J. y Schoeman, C. (2017). Endo-parasites of public-health importance recovered from rodents in the Durban metropolitan area, South Africa. Southern African journal of infectious Diseases, 32(2), 57-66.

Ayulo, V. y Dammert, O. (1947). Survey del parasitismo intestinal de las ratas grises (Mus norvegicus) en la ciudad de Lima. Revista Peruana de Medicina Experimental y Salud Pública, 6(1-4), 7693.
Botero, D. y Restrepo, M. (2012). Parasitosis Humanas. Colombia: Ediciones Corporación para Investigaciones Biológicas (CIB).

Cabrera, R y Mendoza, L. (2001). Heterakis spumosa SCHNEIDER, 1866 (NEMATODA): HETERAKIDAE EN Rattus norvegicus (Rodentia: Muridae) EN ICA, PERÚ. Rev Peru biol. 8(1), 1114.

Chaisiri, K. Chaeychomsi, W. Siruntawineti, J, Ribas, A. Herbreteau, V. y Morand, S. (2018). Gastrointestinal Helminth Infections in Asian House Rats (Rattus tanezumi) from Northern and Northeastern Thailand. Thailandia. I Trop Med Parasitol., 33, 29-32.

Chávez, E. C. and R. Zaldívar. 1967. Zooparasites of livestock in Perú. U.S. Dept. Agríe. Project FG-PE-102. Univ. San Marcos, School of Veto Med. Lima, Perú.

Casana, C. (2018). Prevalencia de Giardia spp. en roedores (Rattus spp.) procedentes de un zoológico de Lima Metropolitana (tesis de pregrado). Universidad Nacional Mayor de San Marcos, Perú.

Coto, H. (2016). Modulo V. Elementos distintivos de Ratas Rattus norvegicus y Rattus rattus. Serie Enfermedades transmisibles. pp. 37-45

De Sotomayor, R. Serrano, E. Tantalean, M. Quispe, M. y Casas, G. (2015). Identificación de Parásitos Gastrointestinales en Ratas de Lima Metropolitana. Rev Inv Vet Perú. 26(2), 273-278.

Escalante, H. (1986). Manual de Técnicas Parasitológicas. Facultad de Ciencias Biológicas - Universidad Nacional de Trujillo. pp. 24-25.

Fagir, M. y El-Rayah, A. (2009). Parasites of the Nile rat in rural and urban regions of Sudan. Sudan. Integrative Zoology.

Fernández, M. (2019). Curso Integral de Plagas Urbanas. Callao.

Garate, I. Jimenez, P. Flores, K. y Espinoza, B. (2011). Registro de Xenopsylla cheopis como hospedero intermediario natural de Hymenolepis diminuta en Lima, Peru. RevPerúbiol. 18(2), 249-252.

Gómez, M. Robles, M. Milano, F. y Navone, G. (2018). Helminth infection levels on Rattus rattus (Rodentia: muridae) from Corrientes city, Argentina. Mastozoologia Neotropical. 
Hancke, D. (2016). La comunidad de helmintos en roedores sinantrópicos de la ciudad de Buenos Aires: su relación con los ensambles de especies hospedadoras y su importancia zoonótica (tesis doctoral). Universidad de Buenos Aires (UBA), Argentina.

Iannacone, J. y Alvariño, L. (2002). Helmintofauna de Rattus rattus (1758) y Rattus norvegicus (Berkenhout, 1769) (Rodentia: Muridae) en el distrito de San Juan de Lurigancho, Lima - Perú. Rev Peru Med Exp Salud Pública. 19(3), 138139.

Ibañez, N. (1966). Nuevo nemátodo parásito de las ratas de Trujillo, Perú. Protospirura chanchanensis sp. n. (Nematoda, Spiruridae). Bol. Chile Parasit. 21,34-37.

Kataranovski, M. Nirkov, I. Belij, S. Popov, A. Petrovic, Z. Gacic, Z y Kataranovski, D. (2011). Intestinal Helminths Infection of rats (Rattus norvegicus) in the Belgrade área (Serbia): The effect of sex, age and habitat. Serbia. Parasitic zoonoses in Europe. Parasite, 18,189-196.

Martínez. R. y M. Tantaleán. (1998). Primer registro en el Perú de Raillietina $(R)$ demerariensis (Cestoda: Taenioidea) en Rattus rattus norvegicus. Rev. peru. biol. 5(1), 65-68.

Mehlhorn, H. (2016). Encyclopedia of Parasitology. Springer, Berlin: Heidelberg.

Mills, J., Childs. J., Ksiazek, T. y Peters, C. (1998). Métodos para trampeo y muestreo de pequeños mamíferos para estudios virológicos. Atlanta: CDC.

MINSA (2003). Manual de procedimientos de laboratorio para el diagnóstico de los parásitos intestinales del hombre. Perú. Serie de Normas Técnicas $N^{\circ}$ 37. pp. 1921

Pakdel, N., Naem, S., Rezaei, F., Chaehchaleh, A. (2013). A survey on helmint hic infect ion mice (Mus musculus) and rats (Rattus norvegicus and Rattus rattus) in kermanshah, Iran. Vet Res Forum, 4, $105-$ 109.

Ramírez, A. (2018). Parásitos gastrointestinales en roedores de la ciudadela "Las Piñas" del Canton Milagro (tesis de pregrado). Universidad de Guayaquil. Ecuador.

Romero, H., Tantaleán, M., Martínez, R., Sáez, G. (2012). Helmintofauna en roedores (Rodentia: Muridae) en mercados del distrito del Rímac. En: Libro de resúmenes del VIII Congreso Peruano de Parasitología. Trujillo

Sarmiento L.; M. Tantaleán y A. Huiza. (1999). Nemátodos parásitos del hombre y de los animales en el Perú. Rev. Pero Parasit. 14, 9-65.

Soulsby, E. (1987). Parasitología y enfermedades parasitarias en los animales domésticos. Organización Panamericana de la Salud. México: Interamericana.

Suarez, J. y Valencia, J. (1986). Ectoparásitos y Helmintos en Ratas y Ratones domésticos (Rattus rattus, Rattus norvegicus, Mus musculus) en la ciudad de Manizales. Universidad de Caldas, Cuba.

Suckow, M. Danneman, P. \& Brayton, C. (2001). The Laboratory Mouse. Washington, D.C. Estados Unidos: CRC.

Tantalean, V. M. (1976). Contribuci6n al conocimiento de los helmintos de vertebrados del Perú. Biota. X, 437-443.

Torres, M. (2017). ¿Son los roedores sinantropicos una amenaza para la salud pública de Yucatán? México. Rev Biomed, 28(28), 179-186.

Vives, N. y Zeledón, R. (1957). Observaciones parasitológicas en ratas de San José, Costa Rica. Rev Bio Trop. 5(2), 173-194.

Zaldívar. S. R. (1991). Zooparásitos de interés veterinario en el Perú. Lima, Perú: Maijosa. 